\title{
Exploring the hidden world of solute atoms, clusters and vacancies in aluminium alloys
}

\author{
John Banhart ${ }^{1 *}$, Zi Yang ${ }^{1}$, Meng Liu ${ }^{1}$, Mazen Madanat ${ }^{1}$, Xingpu Zhang ${ }^{1}$, Qianning Guo ${ }^{1}$, Yong Yan ${ }^{1}$, Andreas Röhsler ${ }^{1}$, \\ Konrad Fricke ${ }^{1}$, Zeqin Liang ${ }^{2}$, David Leyvraz ${ }^{2}$, Armin Hoell ${ }^{1}$, Eike Gericke ${ }^{1}$, Robert Wendt ${ }^{1}$, Chunhui Liu ${ }^{3}$ \\ ${ }^{1}$ Helmholtz-Centre Berlin for Materials and Energy, Hahn-Meitner-Platz 1, 14109 Berlin, Germany \\ ${ }^{2}$ Novelis R\&T Centre, Sierre, Route des Laminoirs 15, 3960 Sierre, Switzerland \\ ${ }^{3}$ State Key Laboratory of High-Performance Complex Manufacturing, Central South University, Changsha 410083, China
}

\begin{abstract}
Precipitation hardening involves solutionising, quenching and annealing steps, the latter often at various temperatures. The phenomena observed in Al-Mg-Si alloys are very complicated and partially not well understood. During and after quenching, solute atoms diffuse through the lattice assisted by vacancies and form atom clusters that gradually grow. These act back onto vacancies, which complicates the situation. We apply positron annihilation techniques in addition to traditional hardness, resistivity and thermal measurements to clarify what happens in various stages of thermal treatment: The quenching process can be divided into a stage of vacancy loss and of precipitation. Very short artificial ageing treatments after heating at different rates show that there is a competition between vacancy losses and cluster formation as the temperature increases. The difference between natural ageing and artificial ageing can be defined based on the importance of excess vacancies. Based on such results the behaviour of "invisible" objects such as vacancies and small clusters can be better understood but some open question remain such as the kinetics of secondary ageing or the details of the negative effect of natural ageing on artificial ageing.
\end{abstract}

\section{Introduction}

Precipitation strengthening in aluminium alloys involves dissolving solute atoms in a matrix at high temperatures, preserving the solid solution by quenching, and ageing the alloy after, during which solute atoms diffuse through the matrix and form clusters, zones and later precipitates terms that are not always defined in the same way in the literature - and increase strength (or hardness). The high vacancy fraction at the solutionising temperature is partially quenched-in and enables diffusion. Knowing vacancy properties is essential for understanding the kinetics of ageing. Thermo-dynamically, age hardening is a non-equilibrium process during which both the vacancy fraction and solute supersaturation change continuously. Various species of atoms may interact and the temperature course can be complex. A variety of different precipitates may form and interact with moving dislocations once there is a mechanical load and thus increase strength.

How complex the scenario is can be seen by considering rate equations for the relative precipitated volume $\alpha$ :

$$
\frac{d \alpha(t)}{d t}=\varphi(1-\alpha(t)) \mathrm{K}(\mathrm{T}) x_{v}(t, T),
$$

where the function $\varphi$ expresses the driving force given by the decreasing solute supersaturation, $\mathrm{K}$ the temperature dependence of precipitation and $x_{v}$ the non-equilibrium vacancy fraction. In thermal equilibrium, $x_{v}$ is a known constant and Eq. (1) can be solved analytically after assuming a reaction function $\varphi$, e.g. the Johnson-Mehl-
Avrami-Kolgomorov (JMAK model [1]. In nonequilibrium, $x_{v}(t, T)$ does not depend on temperature and time in a simple way, but also on $\alpha$, since emerging precipitates interact with vacancies, e.g. trap them. As the interactions between solute atoms, clusters and vacancies are in general not known, Eq. (1) cannot be easily solved.

As precipitation starts with the smallest possible objects, solute-vacancy and solute-solute pairs, knowledge of the earliest stages is crucial to explain subsequent stages in which the final hardened microstructure is formed. However, both vacancies and atomic clusters in such early ageing stages are elusive and experiments meant to gain information are difficult. Positron annihilation spectroscopy (PAS) provides direct access to vacancies, while atom probe tomography (APT) has been used to investigate atomic clusters, but both methods can provide ambiguous results.

In this paper, some of the problems associated with early stages during and shortly after quenching from the solutionising temperature and their influence on subsequent heat treatment are addressed. We start with an overview of the available methods with an emphasis on PAS and then discuss a problem associated with age hardening of Al-Mg-Si alloys. Two attempts to extend currently available methods and two problems associated to Al-Mg-Si alloys, which are waiting to be solved, are presented.

\footnotetext{
* Corresponding author: banhart@helmholtz-berlin.de
} 


\section{Available methods}

\subsection{General overview}

We give a short overview of methods suitable for investigating the small atomic clusters that form during natural ageing (NA) or short artificial ageing (AA). In an earlier paper, some of the methods have already been reviewed [2].

Clustering during NA normally results in changes of mechanical properties. Ageing curves are measured exsitu in various stages of ageing. The main problem is that the exact relationship between cluster parameters and hardness or strength are not known. Fig. 1 illustrates this as it shows TEM images of two samples aged to the same hardness at different temperatures. In (a), fine particles give rise to very little contrast, possibly tiny clusters without an ordered crystal structure. In (b), in contrast, fine particles of monoclinic structure appear, sometimes without even one complete unit cell. This demonstrates that there is no one-to-one relationship between hardness and particle properties.

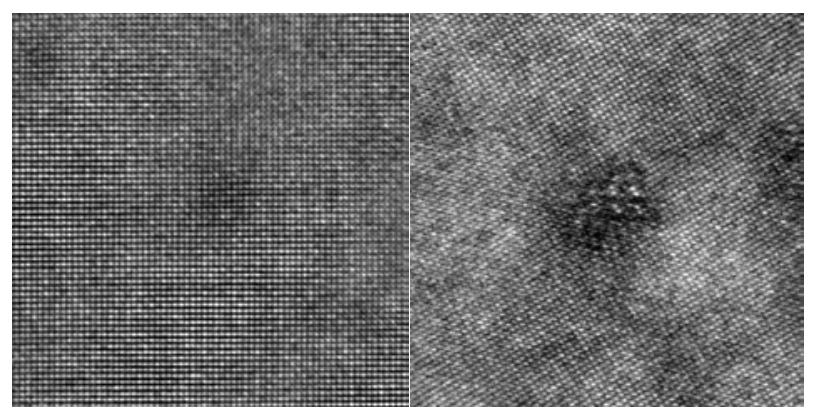

Fig. 1. HR-TEM images of alloy 6014 hardened to $70 \mathrm{HBW}$ 1/10 in two different ways directly after solutionising and quenching, (a) $40 \mathrm{~h}$ at $80^{\circ} \mathrm{C}$, (b) $20 \mathrm{~min}$ at $160^{\circ} \mathrm{C}$. Images recorded without objective aperture display phase contrast.

Electrical resistivity is easy to apply ex-situ [3] or insitu [4]. Sensitivity is high but as for hardness, it is not known precisely how cluster parameters influence resistivity. Vacancies also contribute but it is hard to separate their contribution from that of clusters and solute atoms.

Hardness and resistivity do not vary in the same way during ageing as can be seen by plotting one as a function of the other. It has been argued that an increase of the number of small clusters rather increases resistivity [5], while hardness is influenced more by cluster growth [6].

Thermoelectric power measurements have been used to determine the solute content in the matrix and the influence of precipitates both during NA [7] and AA [8], but to isolate the cluster contribution remains difficult.

$D S C$ clearly shows clustering reactions during isochronal heating [9-11], but interpretations are difficult once various reactions (exothermal, endothermal) overlap. Isothermal DSC has been applied to AA in Al$\mathrm{Mg}-\mathrm{Si}$ alloys [12] and also to NA in Al-Cu-Mg alloys [13], but deconvolution of signals is equally difficult.

Dilatometry has only recently been developed to a method sensitive enough to yield precise information on precipitation in Al-Mg-Si alloys and even the effect of vacancy annihilation during NA has been captured [14].
Small-angle scattering is the classical method to detect nm-sized objects. Neutrons (SANS) yield sufficient contrast to monitor precipitation phenomena in $\mathrm{Al}-\mathrm{Mg}-\mathrm{Si}$ alloys even in-situ [15] during AA but it has not been possible to resolve clusters formed during NA. X-rays (SAXS) have not resolved NA in Al-Mg-Si alloys and as shown below insufficient contrast between clusters and the matrix prevents SAXS from being used successfully.

Table 1. Methods to study early precipitation phenomena in aluminium alloys. Column 'Vac' specifies sensitivity to (mono)vacancies, 'In-situ' whether phenomena can be followed in the same sample during ageing, 'Examples' provides some references for Al-Mg-Si alloys.

\begin{tabular}{llll} 
Observable / Method & Vac ? & $\begin{array}{l}\text { in- } \\
\text { situ? }\end{array}$ & Examples \\
Hardness & no & yes & Sec. 4.1 \\
Strength & no & no & Fig. 4 \\
Electrical resistivity & yes & yes & {$[3]$} \\
Thermoelectric power & no & yes & {$[7]$} \\
Calorimetry & no & yes & {$[9]$, Fig. 6,7 } \\
Dilatometry & yes & yes & {$[14]$} \\
Small-angle scattering & no & yes & Sec. 4.2 \\
X-ray absorption spectr. & no & yes & $($ AA: $[16])$ \\
Nuclear magnetic resonance & no & yes & {$[17]$} \\
Magnetisation & no & yes & {$[18,19]$} \\
Muon spectroscopy & yes & $?$ & {$[20]$} \\
Positron Annihilation & yes & yes & {$[17,21]$} \\
Atom probe tomography & no & no & {$[22-24]$} \\
Electron microscopy (TEM) & no & no & {$[25,26]$} \\
\hline
\end{tabular}

X-ray absorption spectroscopy has been applied to NA in alloys such as Al-Cu [27], while for Al-Mg-Si alloys only AA has been investigated [16].

Nuclear magnetic resonance spectroscopy has been used to follow NA in Al-Mg-Si. Only the ${ }^{25} \mathrm{Mg}$ resonance is usable there but an estimate of the $\mathrm{Mg}$ content in NA clusters as a function of NA time could be obtained [17].

Magnetisation has recently been found to vary during NA. The effects are small $(\leq 0.5 \%$ variation $)$ but measurable and show a similar pattern as positron lifetimes. Si-rich and Mg-rich clusters are thought to give rise to different magnetisation contributions but the exact mechanisms are not yet known $[18,19]$.

Muon spectroscopy relies on measuring the depolarisation rate of the magnetic moments of muons trapped in point defects. As for positron spectroscopy (see Sec. 2.2) it allows one to detect vacancies in alloys [20].

Atom probe tomography provides a direct visualisation of NA clusters and allows for a quantitative analysis. Unfortunately, the results published in the literature are not consistent. The size of clusters hardly seems to evolve during NA [28] and the evolution of their composition has been presented differently. One issue might be the way cluster search algorithms are applied but there might also be a fundamental limitation linked to the trajectories of atoms that prevent very small clusters to be analysed properly [29].

While high-resolution TEM is a remarkable success story when it comes to revealing the smallest details of precipitates in Al-Mg-Si alloys, clusters formed during NA remain elusive and no generally accepted images of NA clusters have been presented. 


\subsection{Positron annihilation lifetime spectroscopy (PALS)}

PALS has been successfully applied to characterise ageing phenomena in various $2 \times x x$ [30], $7 \times x x$ [31] and 6xxx [21] alloys. Its basic principle is simple: Positrons generated during the radioactive decay of ${ }^{22} \mathrm{Na}$ are injected into a sample where they thermalise and start a random diffusional walk. Once they encounter an electron they annihilate. The emitted radiation allows one to measure the lifetime of the positron (PLT). In complex structures such as $\mathrm{Al}-\mathrm{Mg}$-Si alloys containing various different objects, positrons can be trapped by vacancies or precipitates and have a typical lifetime within, or they remain in the bulk Al lattice and decay there. The individual lifetimes have to be extracted from the lifetime spectrum containing signals from many events. The most simple way to view the situation is given in Fig. 2a.
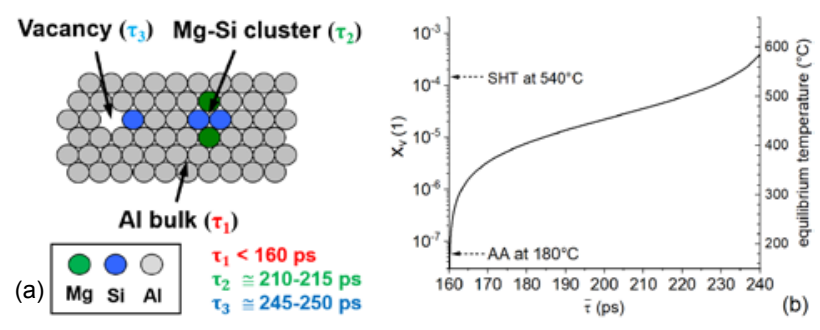

Fig. 2. (a) Schematic of positron annihilation in Al-Mg-Si alloys after NA or short AA including the three typical occurring PLTs. (b) relationship between average PLT and vacancy site fraction in aluminium containing vacancies only and corresponding calculated temperature.

The three associated lifetimes $\left(\tau_{1}, \tau_{2}, \tau_{3}\right)$ are known with only small uncertainty and are given in Fig. 2a. From experimental lifetime spectra, the different contributions have to be extracted by fitting, which is a challenge due to the general problem of de-convoluting decaying exponentials [32], but also because additional corrections such as for background, spectrometer resolution and for annihilation in the positron source itself are necessary [33, 34]. The result of a fit includes the relative intensities of each of the 3 contributions in Fig. $2 a$ and defines an average lifetime:

$\bar{\tau}=I_{1} \tau_{1}+I_{2} \tau_{2}+I_{3} \tau_{3} ; \quad I_{1}+I_{2}+I_{3}=1$.

Such decompositions work only in few favourable cases and require high-count spectra, e.g. Ref. [35]. One can try to treat the traps as one average component characterised by $I_{2+3}$ and $\tau_{2+3}$, thereby reducing the number of fitting parameters or to fit just a single exponential and obtain the one-component lifetime $\tau_{1 C}$, which, however, can deviate from the average lifetime $\bar{\tau}$ if two or more lifetime components are present.

Another problem associated with lifetime decompositions is that more than 2 different traps might co-exist: It is plausible that $\tau_{2}$ varies with cluster composition and therefore there is a range of PLTs. Moreover, the precipitates occurring during AA have different values of $\tau_{2}$ too, e.g. $\tau_{2} \sim 210$ ps for GP zones and $\beta$ " precipitates, but a much higher $\tau_{2} \sim 235 \mathrm{ps}$ for $\beta$ ' precipitates [21]. Finally, solutes attached to vacancies can modify the associated lifetime $\tau_{3}$ [21] and give rise to a distribution of PLTs as well.

If the only trap are vacancies, the two-state trapping model can be used to calculate their site fraction as shown in Fig. 2b. We find: (i) for very low $\bar{\tau}$ just above $160 \mathrm{ps}$ the slope is very large and therefore the vacancy fraction $x_{v}$ cannot be determines, for example, the equilibrium site fraction at $180^{\circ} \mathrm{C}$. (ii) $x_{v}$ values between $5 \times 10^{-7}$ and $5 \times 10^{-4}$ can be measured. (iii) A specific trapping rate for vacancies has to be used in calculating vacancy fractions. It is known within a margin of about a factor of 2 . Unfortunately, the specific trapping rate for other objects such as clusters is not known, so that a calculation in analogy to Fig. $2 \mathrm{~b}$ for the cluster component $\tau_{2}$ cannot be carried out. Clusters in Al-Mg-Si alloys tend to trap positrons efficiently so that $\tau_{2}$ often dominates the positron lifetime spectrum as almost all positrons annihilate within clusters ('saturated trapping').

With these and other facts in mind we can summarise the strengths and weaknesses of PALS in studies of vacancies and clusters in Al-Mg-Si alloys:

+ Sample preparation and measurement is easy,

+ Reproducibility is very good,

+ Highest sensitivity to changes of vacancy/cluster configuration if clustering is not too strong, i.e. average PLT is not between $\sim 200$ and $\sim 220 \mathrm{ps,}$

+ In-situ experiments allow for the detection of small changes even if many clusters are present,

- Lifetime decompositions often not reliable,

- Vacancy and cluster contributions hard to separate,

- Ad-hoc assumptions concerning the nature of positron traps sometimes necessary: lack of calculations of PLTs and specific trapping rates.

In the following, PALS will be applied to gain knowledge about vacancy and cluster evolution in Al-Mg-Si alloys.

\section{Role of excess vacancies}

The terms 'natural ageing' and 'artificial ageing' refer to ageing carried out at 'room temperature' (mostly without defining what this is) and at 'elevated temperature' (usually specified and mostly between $160^{\circ} \mathrm{C}$ and $190^{\circ} \mathrm{C}$ for $6 \mathrm{xxx}$ alloys), but it is not clear what the fundamental difference between the two ageing regimes is.

It has been claimed that there is a transition temperature above which the structure of the precipitates formed during ageing differs from that below and researchers have provided additional evidence [36]. The transition temperature is seen at around $70{ }^{\circ} \mathrm{C}$ and the differences include that clusters formed during NA:

- are richer in $\mathrm{Si}[37,38]$

- contain vacancies $[39,40]$,

- further precipitation during subsequent AA can only take place after cluster dissolution [41, 42].

Fig. 3 shows how hardness evolves at different temperatures. A common feature is the increase in hardness as ageing proceeds. Overageing is seen above $160^{\circ} \mathrm{C}$ and might eventually also occur for lower temperatures, but for NA overageing has never been 
observed. Notably, there is a range, where ageing at lower temperature leads to higher hardness than ageing at higher temperature for the same time, namely for a few hours.

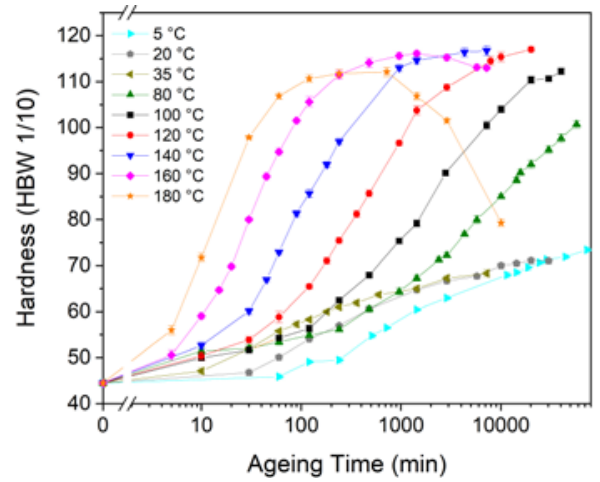

Fig. 3. Isothermal hardening curves for alloy $6014[43,44]$.

This crossover has been used to define a division between NA and AA in terms of strengthening kinetics [45]. To show this clearer the strength reached after an ageing treatment is given as a function of temperature for a fixed ageing time in Fig. 4. The data show two distinct peaks and a minimum in between.

According to the authors the high-temperature peak corresponds to artificial ageing. The more time is available for ageing the higher the achievable strength. Regime III expresses that for a given time higher temperatures give rise to faster ageing and higher strength, but in regime IV this eventually leads to overageing. The same applies to the low-temperature peak (regimes I and II). What is remarkable is the higher strength that can be achieved for ageing at a lower temperature for a given time. For example, ageing for $3 \mathrm{~h}$ at $35^{\circ} \mathrm{C}$ leads to a higher strength than $3 \mathrm{~h}$ at $75^{\circ} \mathrm{C}$ (purple line)

In thermodynamic equilibrium, a reaction at a lower temperature should always be slower than at a higher. A possible explanation would be that at low temperature the type of precipitate formed is different than at higher and that this type is more efficient in increasing strength or that more nuclei are formed at lower temperature. However, as hardening requires non-shearable precipitates of a certain size it is unlikely that this is the only reason. An alternative explanation is that the vacancy fraction has not yet reached thermal equilibrium during NA and that ageing is accelerated by the still available excess vacancies. It has been known for a long time that at 'room temperature' NA can proceed only so fast because the fraction of excess vacancies is many orders of magnitude above the equilibrium value directly after quenching [46]. We therefore claim:

During natural ageing the rate of the strengthening precipitation process is largely determined by excess vacancies, whereas artificial ageing is characterised by precipitation at equilibrium vacancy site fractions.

The 4 regimes in Fig. 4 are then due to:

I. During ageing, excess vacancies anneal out as they go to vacancy sinks but the loss is slow enough to allow for the clustering of a significant amount of solutes. A given vacancy diffuses so slowly that it encounters a sink only after assisting solute atoms in clustering many times, i.e. vacancy loss is not a rate determining factor. At higher temperatures, clustering accelerates, hence the increase in stage I for every given ageing time.

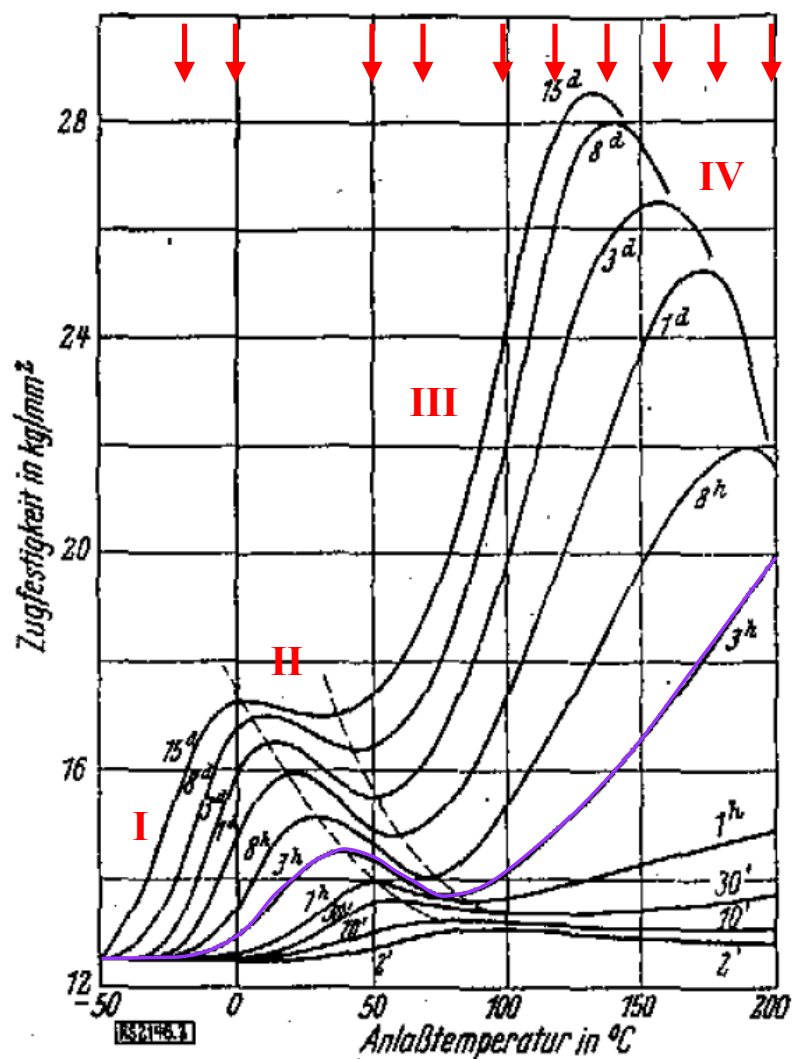

Fig. 4. Tensile strength $\left(1 \mathrm{~kg} / \mathrm{mm}^{2}=9.81 \mathrm{MPa}\right)$ as a function of ageing temperature for 10 different ageing times ranging from 2 min (2') to 15 days $\left(15^{\mathrm{d}}\right)$. No individual data points are given, just interpolated lines based on data at the measurement temperatures marked by vertical arrows at the top. Alloy Al-0.4 $\mathrm{Mg}-0.55 \mathrm{Si}-0.2 \mathrm{Fe}$ (probably wt.\%) was solutionised for $1 \mathrm{~h}$ at $530^{\circ} \mathrm{C}$ and water quenched after. After immersion into oil or a salt bath the final ageing temperature was reached in less than $1 \min [45]$.

II. After exceeding a certain temperature, vacancy losses during a given period of ageing start influencing ageing kinetics. When ageing for $8 \mathrm{~h}$, for example, the vacancies are still largely preserved at $20^{\circ} \mathrm{C}$, but when moving to $80^{\circ} \mathrm{C}$, vacancy concentration drops so quickly during ageing that clustering kinetics is retarded despite the in general much faster solute diffusion.

III. Once vacancy concentration has dropped to an equilibrium value shortly after the onset of artificial ageing, kinetics are mainly determined by temperature. For higher temperatures, diffusion under equilibrium conditions produces more precipitates in a given time.

IV. For very high temperatures, fewer precipitates form and over-ageing sets in.

Before presenting experimental evidence for this concept we apply a simple model. First, the diffusion of a $\mathrm{Al}$ or solute atom is represented by a product of vacancy fraction and a $\mathrm{Al} /$ solute migration enthalpy $H^{m}$ related term 


$$
D(T, t) \sim x_{v}(T, t) e^{-H^{m} / k T} .
$$

Unlike in the definition of an equilibrium diffusion coefficient, $x_{v}$ is a non-equilibrium value here and depends on time and thermal history. Its equilibrium value after a long time is $x_{v}(T)=\exp \left(S / k-H_{v}^{f} / k T\right)$. In equilibrium, the activation energy of self diffusion is $Q_{\mathrm{Al}}^{d}=H_{v}^{f}+H_{\mathrm{Al}}^{m}$, where the vacancy formation enthalpy $H_{v}^{f}$ and the migration enthalpy of $\mathrm{Al}, H_{A l}^{m}$ can be measured or calculated. $H_{A l}^{m}$ also describes the migration of vacancies. We use values, $S / k=0.7, H_{v}^{f}=0.665 \mathrm{eV}[47$, 48], and $H_{A l}^{m}=0.655 \mathrm{eV}$ [48]. Published diffusion data for impurities in $\mathrm{Al}$ show a large variance and none are available for 'room temperature'. Especially the breakdown of diffusion values into pre-factor $D_{0}$ and activation energy $Q$ is notoriously unreliable. Therefore, we partially rely on calculated data here. $H_{S i}^{m}$ for $\mathrm{Si}$ is $0.48 \mathrm{eV}$ as calculated from $Q_{S i}^{d}=1.15 \mathrm{eV}$ [49]. For $\mathrm{Mg}$, this value is higher by $0.12 \mathrm{eV}$, but note that Ref. [48] quotes almost swapped values for $\mathrm{Mg}$ and $\mathrm{Si}$.

Using a model of vacancy annihilation allows one to calculate $x_{v}(t, T)$ after quenching from $5400^{\circ} \mathrm{C}$ and during subsequent isothermal ageing [50] and to apply Eq. (2). Diffusing solute atoms reach other solutes and form clusters. The diffusion range of a solute atom in time $t$ is

$$
\bar{r}=\sqrt{6 D_{s}(T, t) t} .
$$

We assume that in a given time interval $\Delta t$ a solute leads to a precipitation activity $\Delta \alpha$ proportional to the distance $\Delta \bar{r}$ covered and obtain for the precipitation rate

$$
\frac{\mathrm{d} \alpha}{\mathrm{d} t}=A \frac{\mathrm{d} \bar{r}}{\mathrm{~d} t}=\frac{A}{2 \sqrt{t}} \sqrt{6 D(T, t)},
$$

with $A$ a constant. As precipitation proceeds, solute atoms are consumed and the driving force decreases. $\frac{\mathrm{d} \alpha}{\mathrm{d} t}$ in Eq. (4) has to be multiplied by a solute supersaturation factor $\varphi$ decreasing with $\alpha$ to prevent $\alpha$ from increasing indefinitely (in analogy to Eq. (1) in Ref. [4]). As we do not know the functional relationship between $\varphi$, the driving force, and solute concentration we write

$$
\varphi(\alpha)=(1-\alpha)
$$

Eq. (4) multiplied with Eq. (5) and using Eq. (2) is now equivalent to the general rate equation Eq. (1) and ensures $\alpha \in[0,1]$. Eq. (4) is just a special case for $n=1$ of the generalised JMAK equation [1]:

$$
\varphi(\alpha)=n(1-\alpha)[-\ln (1-\alpha)]^{1-\frac{1}{n}}
$$

We now numerically integrate $\frac{\mathrm{d} \alpha}{\mathrm{d} t} \varphi(\alpha)$ using the calculated $x_{v}(T, t)$ and obtain $\alpha(t)$. It is experimentally known that the precipitated volume is proportional to hardness or strength during NA $[44,51]$ and we extend this to AA as we are only interested in trends, i.e. write $\Delta S=\alpha$, with $\Delta S$ the relative strength increase. The adjustable constant $A$ in Eq. 3 is chosen such that $\Delta S$ during NA is about $1 / 3$ of $\Delta S$ during $\mathrm{AA}$, which matches reality, see Fig. 3.
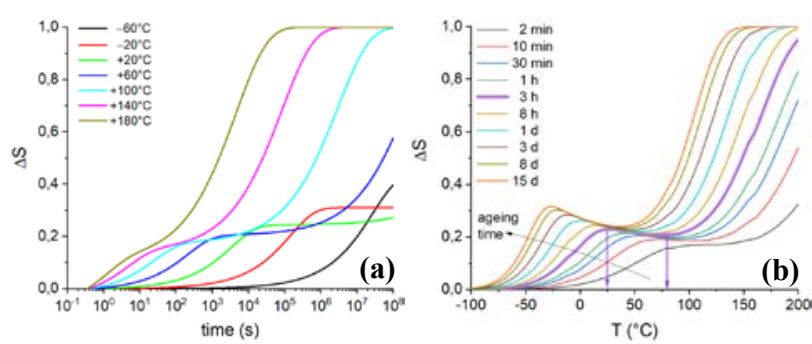

Fig. 5. Calculated strengthening as a function of a) time for fixed $\mathrm{T}$ or $\mathrm{b}$ ) of $\mathrm{T}$ for fixed times (same as in Fig. 4). Model parameters: dislocation density $3 \times 10^{12} \mathrm{~m}^{-2}$, jog spacing 0.02 , grain diameter $50 \mu \mathrm{m}, H_{v}^{f}=0.665 \mathrm{eV}, S / k=0.7, H_{A l}^{m}=0.655 \mathrm{eV}$, $H_{A l}^{m}=0.48 \mathrm{eV}$ (see Ref. [50] for description of model)

Fig. 5a features a clear two-stage ageing behaviour for the higher temperatures. Fig. $5 \mathrm{~b}$ represents the experimental hardening curves in Fig. 4 except for the over-ageing regime IV that is not included in the model. As an example, ageing for $3 \mathrm{~h}$ (purple curve) leads to a maximum of precipitation for $25^{\circ} \mathrm{C}$ and a subsequent minimum at $80^{\circ} \mathrm{C}$, which is what the experiment shows as well, see purple curve in Fig. 4.

Choosing values $n \neq 1$ in Eq. (5a) or the function proposed in Eq. (4) of Ref. [4] does not have a pronounced influence on the principle course in Fig. 4. This is because the existence of the decrease in stage II is due to the fact that $H_{S i}^{m}<H_{A l}^{m}$ has been adopted for $\mathrm{Si}$ atoms (see comments above). A lower ageing temperature can lead to more precipitation due to an excess vacancy concentration that exceeds that at a higher temperature by a larger factor than the solute diffusion rate is lower. In other words, the $H_{A l}^{f m}=0.656 \mathrm{eV}$, which determines the kinetics of vacancy migration to sinks, penalizes ageing at higher temperature more than the gain in solute migration (and precipitation) rate given by $H_{S i}^{m}=0.48 \mathrm{eV}$. Thus, this simple kinetic model that ignores details of precipitate nucleation and type explains the behaviour of ageing at different temperatures surprisingly well and underlines that excess vacancies cause strong ageing at natural ageing temperatures. Thus, a main feature distinguishing NA and AA is the role of vacancies and the transition temperature is not fixed but depends on ageing time.

We now refer to 2 experiments to show that at the usual artificial ageing temperature of $180^{\circ} \mathrm{C}$ ageing kinetics are determined by the equilibrium vacancy concentration only.

In the first, DSC is used to visualise the exothermic clustering peak that is observed when $6 \mathrm{xxx}$ alloys are heated [10]. Fig. 6a shows the part of the DSC trace in which this clustering peak occurs, namely from $30^{\circ} \mathrm{C}$ to $130{ }^{\circ} \mathrm{C}$. If samples are heat treated at $180{ }^{\circ} \mathrm{C}$ for up to $30 \mathrm{~s}$ before DSC is run, this peak disappears as expressed by the relative area of the clustering peak in Fig. $6 b$.

There are two possible explanations for the disappearance of the peak in such a short time: Due to ageing before DSC (i) solutes precipitate within seconds, so that during DSC no more clusters can be formed due to a low solute level; (ii) the vacancy concentration drops to such a low value so that during ensuing DSC kinetics are slowed down so much that no more precipitation takes place in the 10 minutes of heating from $30^{\circ} \mathrm{C}$ to $130^{\circ} \mathrm{C}$. 

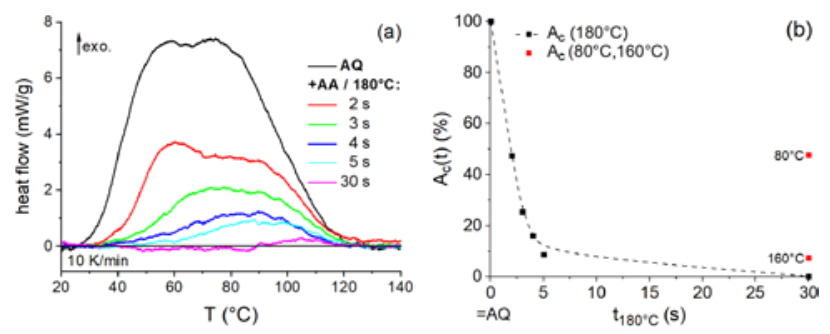

Fig. 6. (a) Clustering peak observed by DSC directly after quenching (AQ) and after an additional short treatment at various temperatures in an oil bath. (b) Areas $\boldsymbol{A}_{\boldsymbol{c}}$ of clustering peak with respect to the AQ state as a function of time. Alloy Al-0.6Mg-0.8Si (wt.\%). Data taken from Ref. [52].

As heating for a few seconds at $180^{\circ} \mathrm{C}$ does not lead to any measurable hardening (just $2 \mathrm{HBW}$ in $1 \mathrm{~min}$, see Fig. 9) and not to more than a slight change of resistivity, which can be negative or positive [53], option (i) is unlikely. This is different for NA performed before DSC which also brings down the area of the DSC peak to about $50 \%$ after $1 \mathrm{~d}$, see Fig. 7 , but the associated hardness increase from 44 to $65 \mathrm{HV}$ is notable (Fig. 3), which shows that in this case (i) is significant. Moreover, the low-temperature part of the clustering part is selectively eliminated here, whereas short AA decreases the entire profile of the DSC clustering peak equally as vacancies are removed and kinetics are slowed down globally while the solute configuration remains largely unchanged.

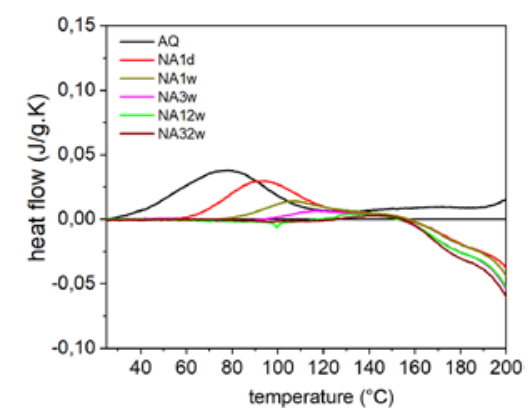

Fig. 7. DSC traces of alloy 6014 directly after quenching and after NA at $20^{\circ} \mathrm{C}$ for different times.

A positron lifetime experiment provides further insights. Al-Mg-Si alloys were subjected to ageing treatments directly after quenching as short as $1 \mathrm{~s}$ and positron lifetime (PLT) measured directly after.
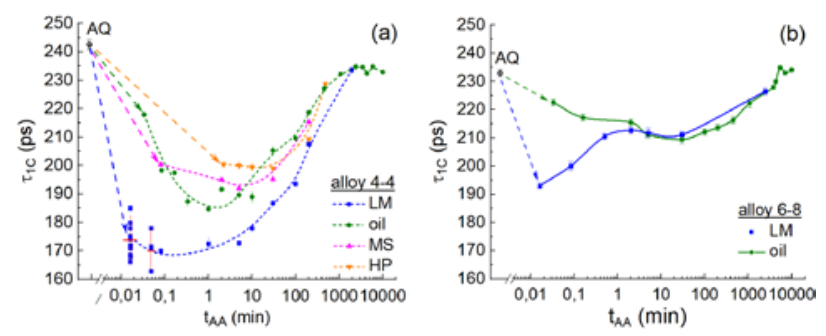

Fig. 8. Positron lifetime measured after solutionising, quenching and artificial ageing at $180{ }^{\circ} \mathrm{C}$ of a) alloy $\mathrm{Al}-0.4 \mathrm{Mg}-0.4 \mathrm{Mg}$ and b) $\mathrm{Al}-0.6 \mathrm{Mg}-0.8 \mathrm{Si}$ (wt.\%) in different media [53].

In Fig. 8a, the initially high positron lifetime of a lean alloy directly after quenching ('AQ') is associated to a high vacancy fraction. It is reduced to a very low level by
AA just for $1 \mathrm{~s}$. Values close to $160 \mathrm{ps}$ - defect free $\mathrm{Al}-$ imply that most of the vacancies have been eliminated by such a short treatment at $180{ }^{\circ} \mathrm{C}$, see Fig. $2 \mathrm{~b}$. The degree of lifetime reduction depends on the heat bath used. It is most pronounced for liquid Bi-Sn alloy where the final temperature is reached almost instantaneously. We can use the lowest value observed in Fig. 8 to estimate the vacancy fraction after short AA by applying the two-state trapping model and assuming the absence of any clusters and obtain $x_{v}=2.5 \times 10^{-7}[53]$. This is just 3 times the equilibrium vacancy fraction of $180{ }^{\circ} \mathrm{C}$ and possibly the real $x_{v}$ was even lower if some clusters were present.

When ageing in molten salt ('MS'), oil or on a heating plate ('HP', where temperature is ramped up at $3 \mathrm{~K} / \mathrm{s}$ ) PLT reduction by ageing at $180{ }^{\circ} \mathrm{C}$ is less pronounced. This is because slower heating allows the alloy to be exposed more to the low-temperature range where clusters form and add a lifetime component of $\sim 215$ ps to the spectrum.

In the alloy richer in solute, see Fig. $8 b$, the positron lifetime reductions are less pronounced with $190 \mathrm{ps}$ the lowest value measured in LM and slower heating leads to an even shallower minimum than in the leaner alloy. This is either due to the presence of more clusters or more vacancies than in Fig. 8a. Clusters may have formed already during quenching and further grown in the short time passing through low-temperature regimes when heating up the alloy at a reduced rate. The mechanism behind this could be that clusters formed in large numbers assisted by excess vacancies help preventing annihilation of these vacancies for a little while during heating, which in turn supports further clustering [53]. For very long ageing times the PLT increases to high values up to $235 \mathrm{ps}$. This is due to a high PLT in the $\beta$ ' precipitates that form in late stage of (over-)ageing.

There is another argument against the existence of excess vacancies during artificial ageing for an extended period of, say, minutes. Hardening directly after solutionising and quenching for up to $10 \mathrm{~min}$ would be initially very fast and then level off in this case. In reality this is not observed, see Fig. 9 and the hardening rate is rather constant.

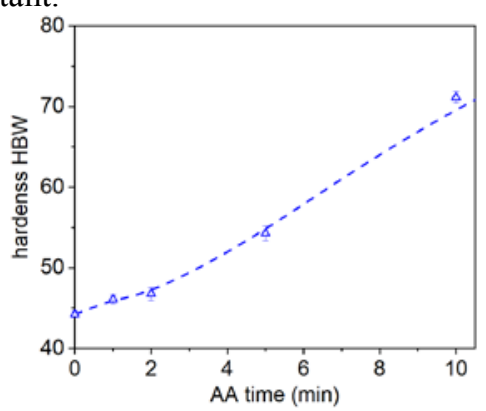

Fig. 9. Hardness of alloy 6014 after short artificial ageing at $180^{\circ} \mathrm{C}$.

Positron lifetime spectroscopy is most useful in states where lifetimes are very high or very low. This is illustrated in Fig. 10, where information about the vacancy and cluster evolution during slow quenching is obtained by allowing samples to cool to a given temperature after solutionising and then interrupting by a fast quench. The positron lifetimes clearly change in 2 stages. (i) First, a continuous drop down to values around 
$170 \mathrm{ps}$ for both cooling rates. Such values point at very low fractions of both vacancies and clusters, namely vacancy fractions $\leq 2 \times 10^{-6}$ in the absence of clusters, see Fig. 2b, and even lower fractions if clusters form during cooling. (ii) If slow cooling is interrupted at temperatures lower than $200{ }^{\circ} \mathrm{C}$, the resulting PLT is higher again, reaching $\sim 215 \mathrm{ps}$ for uninterrupted slow quenching. As the vacancy site fraction cannot increase from its minimum achieved at $200{ }^{\circ} \mathrm{C}$, the PLT increase below $200{ }^{\circ} \mathrm{C}$ must be entirely due to clustering. Therefore, the quenching process can be divided to 2 stages: predominantly vacancy losses down to $200{ }^{\circ} \mathrm{C}$ and clustering after.

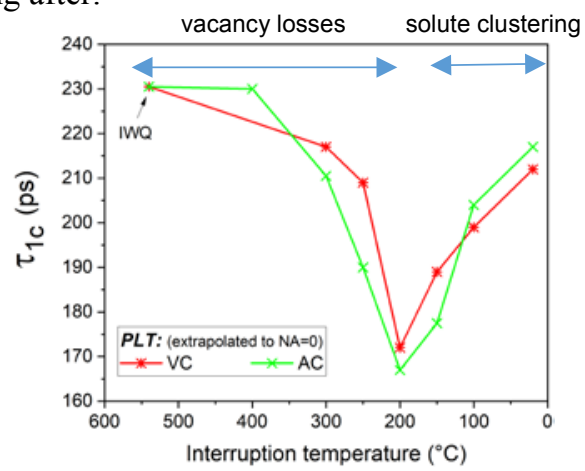

Fig. 10. Positron lifetime in alloy 6014 samples solutionised and cooled at $27 \mathrm{~K} / \mathrm{s}(\mathrm{VC})$ and $5 \mathrm{~K} / \mathrm{s}(\mathrm{AC})$ down to a target temperature, after which fast quenching to $20^{\circ} \mathrm{C}$ in ice water largely preserves the resulting microstructure. The first point corresponds to uninterrupted fast quenching, the last to uninterrupted slow quenching.

Fig. 11 shows another experiment with an interrupted temperature course. Here, as-quenched samples were heated to a target temperature at three different rates, after which heating was interrupted by fast quenching. Directly after quenching, PLT is high, indicating predominance of vacancies. After heating to $60{ }^{\circ} \mathrm{C}$, the PLT is almost at the level of NA for $1 \mathrm{~h}$, i.e. clusters dominate the signal. A weak vacancy-related signal survives up to $100^{\circ} \mathrm{C}$, for the highest heating rate. From the previous discussion and Fig. 6a, we know that during linear heating clusters occur and that this is driven by excess vacancies. In this case, however, PALS cannot provide clear information about the fraction of vacancies due to the predominance of clusters. After heating from $140^{\circ} \mathrm{C}$ to $220^{\circ} \mathrm{C}$, a minimum is found that might be due to the decrease of cluster site fraction and the development of a coarser distribution of precipitates. The final increase corresponds to the increase in Fig. 8 for long ageing times caused by formation of $\beta$, precipitates.

We conclude this section by noting that non-equilibrium vacancies have an important influence on ageing and that a combination of PALS with DSC and hardness measurements allows one to elucidate the co-evolutions of clusters and precipitates both during isothermal ageing and linear heating. However, ambiguities still exist, which is why new experimental options should be evaluated. This will be briefly review in the following section.

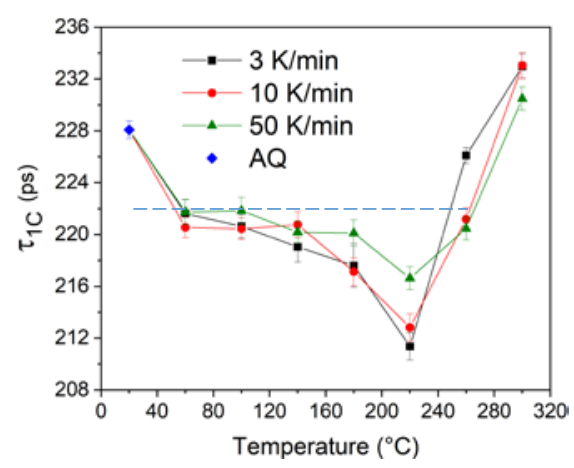

Fig. 11. Positron lifetimes after solutionising and quenching and subsequent linear heating at 3 different heating rates from $20^{\circ} \mathrm{C}$ to a selected end temperature. Horizontal line marks approximate PLT level after $1 \mathrm{~h}$ of NA. Alloy 6014, [44].

\section{Extension of characterisation methods}

\subsection{In-situ NA hardness measurements}

Hardness measurements provide an indirect measure for clustering or precipitation. Although their interpretation is not always straight-forward they are often applied as a first orientation. The basic problem is the labour intensive procedure when carried out manually so that often ageing curves consist only of a small number of measurements, for example $14 \mathrm{NA}$ times and 10 hardness measurements for each time of the NA curve in Ref. [2] where two or three distinct linear stages on the logarithmic time scale were postulated with a transition time between 50 and $80 \mathrm{~min}$. By performing automated micro-hardness measurements one can largely increase the number of measurements and obtain a clearer indication whether hardness evolves in stages, as seen in Fig. 12, where the existence of two stages with a transition around $1 \mathrm{~h}$ of NA is seen much more clearly than in Ref. [2].

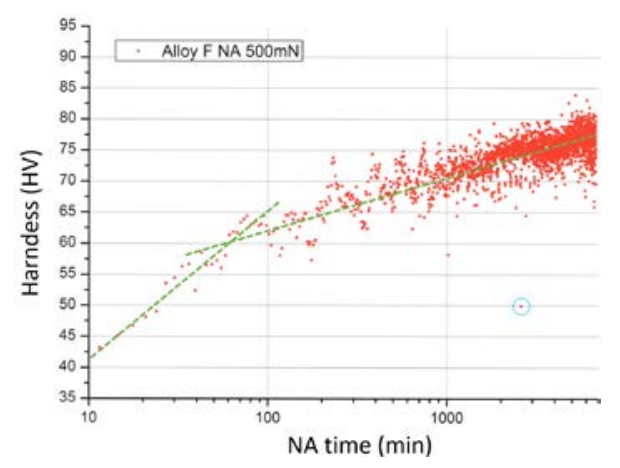

Fig. 12. In-situ micro-Vickers hardness measurement on alloy Al-0.6Mg-0.8Si (wt.\%) carried out with a Fisher 'Picodentor HM500' during NA following solutionising and quenching. One hardness indentation every 3 minutes was automatically obtained and in total 2195 data points acquired over a period of $110 \mathrm{~h}$. Force was $0.5 \mathrm{~N}$. The broken green lines are linear fits to the data for $<100 \mathrm{~min}$ and $>100 \mathrm{~min}$ NA time. The broken circle is an example for irregular results removed from the data set.

\subsection{In-situ SAXS measurements}

SAXS is expected to yield only small signals from $\mathrm{Mg}$ and $\mathrm{Si}$ rich clusters in Al-Mg-Si alloys due to the small difference in scattering length between $\mathrm{Al}, \mathrm{Mg}$ and $\mathrm{Si}$ that is proportional to the number of electrons in an atomic 
species. The scattering length of almost equal quantities of $\mathrm{Mg}$ and $\mathrm{Si}$ in clusters nearly cancels out compared to Al [2]. Thus, the contrast between the clusters and the matrix is only caused by differences of the mass density of both phases. Still, we attempted to overcome the difficulties by applying an in-situ experiment on a solutionised and quenched sample in which all conditions remained constant so that any change during NA could be ascribed to clustering phenomena.

Alloy Al-0.6Mg-0.8Si (wt.\%) was polished to a $60 \mu \mathrm{m}$-thick platelet, solutionised at $540{ }^{\circ} \mathrm{C}$ between two protective titanium plates and water quenched. Within $90 \mathrm{~s}$ the sample was transferred to a transport box filled with liquid nitrogen. SAXS was measured using the HZB Anomalous SAXS instrument [54] at the four-crystal monochromator (FCM) beamline of the PTB at the BESSY-II storage ring using an in-vacuum Pilatus $1 \mathrm{M}$ detector [55] at a X-ray energy of $(8980.5 \pm 2.0) \mathrm{eV}$. The sample was mounted onto a holder in liquid nitrogen and rapidly transferred to the sample chamber. After reaching $4 \times 10^{-4}$ mbar during evacuation, the measurement was started, initially at a temperature of $3{ }^{\circ} \mathrm{C}$ that eventually rose to $25^{\circ} \mathrm{C}$ during the SAXS experiment (see Fig. 13c, blue line).

SAXS scattering images were measured at five different spots on the alloy platelet. The X-ray transmission and sample thickness (calculated from the transmission coefficient) varied slightly between the spots on the platelet but were found to be constant over time. Acquisition times of initially 30 seconds and later 120 and 300 seconds were used per scattering image. In further data treatment, all images were azimuthally averaged and normalized to absolute scattering cross-sections. The instrumental scattering background was subtracted.
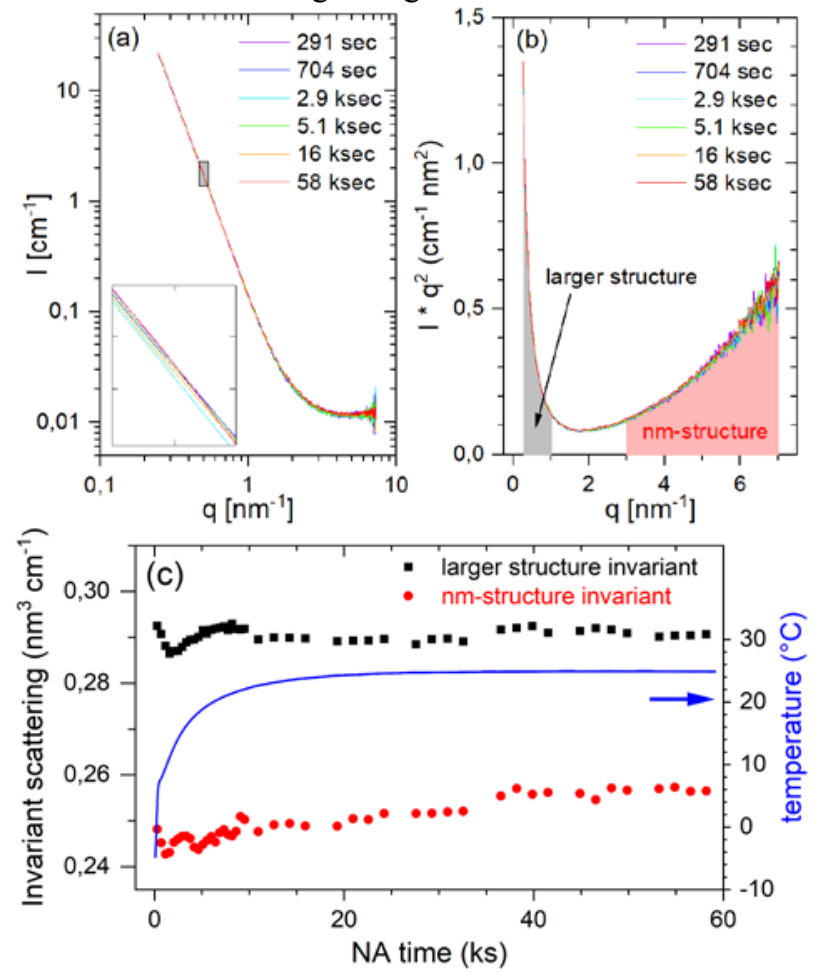

Fig. 13. SAXS results on a logarithmic scale (a) and as a Kratky plot (b). NA time dependence of the two integrals marked by a grey and a red area in the Kratky plot are shown in (c).
The SAXS curves change only slightly over time, see Fig. 13a. The inset provides a close up of the SAXS curves in the q-range $(0.48-0.50) \mathrm{nm}^{-1}$ and indicate a nearly nonrecognizable change during the in-situ experiment. The scattering intensity is plotted against the value of momentum transfer (q). The q-value is related to the scattering angle $(2 \theta)$ by $q=4 \pi / \lambda \sin (\theta)$, with $\lambda$ the $X$ ray wavelength). In Fig. 13b, the Kratky plot (I· $\mathrm{q}^{2}$ vs. q) of the SAXS curves is shown. The integral of $\mathrm{I} \cdot \mathrm{q}^{2}$ over $\mathrm{q}$ is the so-called invariant of scattering and allows for the calculation of a particle volume fraction in two-phase systems. The integral between q-values of 3 and $7 \mathrm{~nm}^{-1}$ depends on very small nano-structures ( 0.9 to $2 \mathrm{~nm}$ size) (Fig. 13b, red area). In contrast, the integral between the smaller q-values of 0.26 and $1 \mathrm{~nm}^{-1}$ depends on structures larger than $6 \mathrm{~nm}$ (Fig. 13b, grey area). In Fig. 13c, both integrals are plotted against aging time as black squares (larger-structural invariant) and red circles (nanostructural invariant). Only the latter shows a change with NA time, which is a weak signature of clustering.

This experiment confirms that SAXS, although it captured a faint signature of clustering is not capable of yielding detailed data in pure ternary $\mathrm{Al}-\mathrm{Mg}-\mathrm{Si}$ alloys. The situation would be different in quaternary systems containing elements that scatter stronger, for example $\mathrm{Cu}$. By carrying out SAXS at various energies slightly below the absorption edges of the elements involved here (e.g. $\mathrm{Si}$ and $\mathrm{Cu}$ ) one could obtain element specific scattering curves. Such ASAXS experiments could reveal the structure of $\mathrm{Si}$ or $\mathrm{Cu}$ containing clusters when carried out near the Si K-edge $(1.84 \mathrm{keV})$ or Cu K-edge $(8.98 \mathrm{keV})$.

\section{Open questions}

\subsection{Natural secondary ageing (NSA)}

Unlike (primary) NA that is carried out after solutionising and quenching, natural secondary ageing (NSA) takes place after an underageing treatment (UA) at AA temperatures short enough to still allow for ageing phenomena to occur during subsequent NA. Such treatments have been investigated with an application background in mind (called T6I4 and T6I6 [56]) but the phenomena during NSA are also worth being studied because UA represents a state of high solute supersaturation but low vacancy fraction and NSA provides indirect information about ageing phenomena during UA.

The kinetics of NSA show some peculiarities that are not easy to understand. Fig. 14 shows the rate of electrical resistivity change during NA/NSA after different UA treatments (including no UA). As expected, the highest rate is found directly after solutionising and quenching as the vacancy and solute supersaturation are the highest. During NA the rate continuously decreases. This is also the case after UA with the notable exception of alloys containing tin. In these alloys, the rate increases by one order of magnitude after 1 week of NSA and peaks, see arrows. Such an increase is not explainable in terms of the picture of a rate proportional to excess vacancy fraction as given by Eq. (1). 


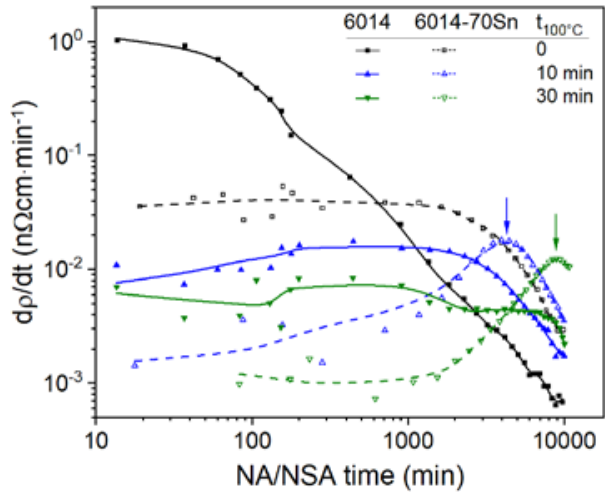

Fig. 14. Rate of electrical resistivity increase after solutionising and quenching as a function of NA/NSA time in 6014 alloys without and with $70 \mathrm{ppm}$ Sn addition [57].

Hardening during NSA after short UA at $180{ }^{\circ} \mathrm{C}$ is also seen to yield unexpected results, see Fig. 15. Here, NSA directly after quenching leads to hardening from 42 to 70 $\mathrm{HV}$ within one year. After UA for up to $5 \mathrm{~min}$, the total NSA course is only slightly changed, most notably in the first $100 \mathrm{~min}$. One would expect that removal of most of the vacancies from the matrix by short exposure to $180^{\circ} \mathrm{C}$, see discussion above and Fig. 6, would notably slow down NSA. However, there is a mechanism that seems to equalise conditions, for example by trapping most excess vacancies in clusters, so that after $100 \mathrm{~min}$ of NSA the slope of hardness increase is very similar for AA times up to $5 \mathrm{~min}$. Only for much longer UA times the hardness increase during UA has set in and the hardness during NSA is largely constant.

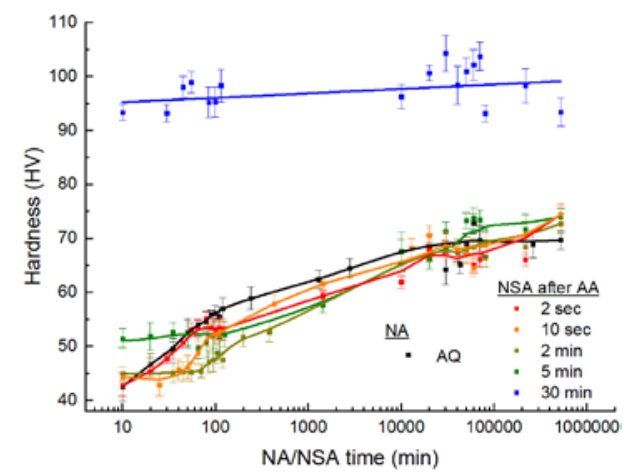

Fig. 15. Hardness evolution during NA/NSA at $20^{\circ} \mathrm{C}$ after UA for $2 \mathrm{~s}$ up to $30 \mathrm{~min}$.

\subsection{Reversal of negative effect of natural ageing}

In solute-rich $\mathrm{Al}-\mathrm{Mg}-\mathrm{Si}$ alloys, artificial ageing is compromised by prior natural ageing. Solute and vacancies are locked up in clusters or clusters might have to dissolve before strengthening $\beta$ " particles can be formed because direct conversion is not possible. The relative importance for each mechanism is not known but an even bigger surprise is that the negative effect does not continuously build up with longer NA time but sometimes is reversed. Fig. 16 demonstrates this for the alloy Al$0.6 \mathrm{Mg}-0,8 \mathrm{Si}$ (wt.\%). For intermediate AA times $(2 \mathrm{~h}, 4 \mathrm{~h}$, $8 \mathrm{~h}$ ), NA up to 1 day increasingy reduces AA response (arrow 1), but for very long times the negative influence is partially reversed (arrow 2), after which it starts to be restored again (arrow 3). For very short AA (2, 5 min), short NA can increase AA response (arrow 4), but for very long NA, AA up to 30 min decreases hardness (arrow 5) [52]. Two papers have also discussed these effect $[58,59]$. The clusters formed in different stages of NA possibly have different stabilities and the ones corresponding to the largest negative effect are more difficult to dissolve, but the structural reasons for this behaviour are not clear. It has been observed that cluster dissolution, which is a prerequisite for fast $\mathrm{AA}$, depends on the alloy composition [42], which could be a key for a consistent explanation, but further studies are needed before one can claim to understand and eventually control the effect. Table 2 lists some references of work containing data showing some of the effects of Fig. 16.

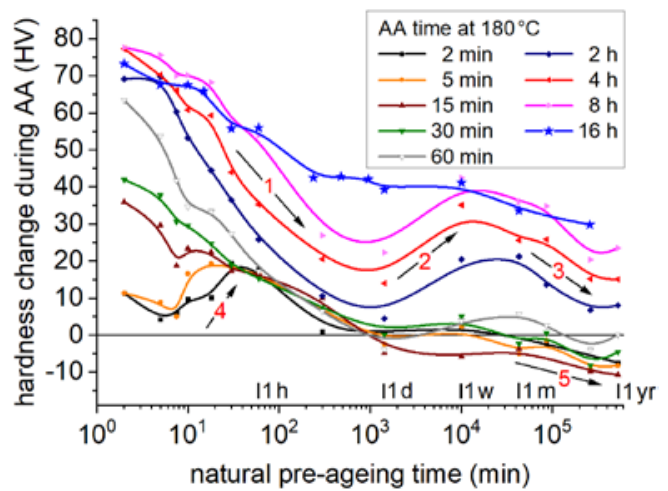

Fig. 16. Hardening response of alloy $\mathrm{Al}-0.6 \mathrm{Mg}-0.8 \mathrm{Si}$ (wt.\%) during AA at $180^{\circ} \mathrm{C}$ for different times after natural pre-ageing (calculated from data of Refs. [52, 60]). Arrows: effects discussed, 1: establishment of negative effect of NA, 2: partial reversion of negative effect, partial restoration of negative effect, 4: positive effect of short NA for short AA treatments, 5: cluster dissolution effect.

Table 2. Studies reporting reversal and restoration of the negative effect of NA on AA.

\begin{tabular}{lll}
$\begin{array}{l}\text { Phenomenon } \\
\text { observed }\end{array}$ & $\begin{array}{l}\text { This work } \\
\text { (from [52, 60]) }\end{array}$ & References \\
\hline $\begin{array}{l}\text { Partial reversal } \\
\text { (arrow 2) }\end{array}$ & yes & {$[58,59,61-66]$} \\
$\begin{array}{l}\text { Partial restoration } \\
\text { (arrow 3) }\end{array}$ & yes & {$[59,61,66]$} \\
$\begin{array}{l}\text { Initial increase } \\
\text { (arrow 4) }\end{array}$ & only for short AA & {$[58,66,67]$} \\
\hline
\end{tabular}

\section{Conclusions}

Vacancies play a key role in determining the kinetics of precipitation in aluminium alloys. A high excess vacancy fraction during ageing at low temperatures is crucial for the fast hardening observed there. Positron annihilation lifetime spectroscopy provides direct evidence and quantitative estimates of the evolution of vacancy fraction in $\mathrm{Al}-\mathrm{Mg}-\mathrm{Si}$ alloys during quenching and short artificial ageing. Due to the limitations of the currently known methods, new developments are needed for deeper insights into the subtle problems of early ageing phenomena. 
Acknowledgements: We acknowledge help in the analysis of SAXS data by our student Sarah Flöter.

\section{References}

[1] M.J. Starink, Int Mater Rev 49, 191 (2004)

[2] J. Banhart, C.S.T. Chang, Z.Q. Liang, et al., Adv Eng Mater 12, 559 (2010)

[3] C. Panseri, T. Federighi, J I Met 94, 99 (1966)

[4] M. Madanat, M. Liu, J. Banhart, Acta Mater 159, 163 (2018)

[5] S. Esmaeili, D. Vaumousse, M.W. Zandbergen, et al., Philos Mag 87, (2007)

[6] S.N. Kim, J.H. Kim, H. Tezuka, et al., Mater Trans 54, 297 (2013)

[7] D. Dafir, G. Guichon, R. Borrelly, et al., Mater Sci Engnr A 144, 311 (1991)

[8] V. Massardier, T. Epicier, P. Merle, Acta Mater 48, 2911 (2000)

[9] A.K. Gupta, D.J. Lloyd, Metall Mater Trans A 30, 879 (1999)

[10] I. Dutta, S.M. Allen, J Mater Sci Lett 10, 323 (1991)

[11] C.S.T. Chang, J. Banhart, Metall Mater Trans A 42A, $1960(2011)$

[12] S. Esmaeili, D.J. Lloyd, W.J. Poole, Acta Mater 51, 3467 (2003)

[13] A.M. Zahra, C.Y. Zahra, C. Alfonso, et al., Scripta Mater 39, (1998)

[14] M. Luckabauer, E. Hengge, G. Klinser, et al., in: Magnesium Technology 2017, K.N. Solanki (Ed.) The Minerals, Metals, \& Materials Society, 2017, pp. 669-674

[15] C.S.T. Chang, F. De Geuser, J. Banhart, J Appl Crystallogr 48, 455 (2015)

[16] H. Adachi, H. Nakanishi, M. Asano, J. Japan Inst. Light Met. 65, 411 (2015) [in Japanese]

[17] M.D.H. Lay, H.S. Zurob, C.R. Hutchinson, et al., Metall Mater Trans A 43A, 4507 (2012)

[18] K. Nishimura, K. Matsuda, T. Namiki, et al., Mater Trans 56, 1307 (2015)

[19] K. Nishimura, K. Matsuda, Q.K. Lei, et al., Mater Trans 57, 627 (2016)

[20] S. Wenner, C.D. Marioara, K. Nishimura, et al., Metall Mater Trans A 50A, 3446 (2019)

[21] J. Banhart, M.D.H. Lay, C.S.T. Chang, et al., Phys Rev B 83, 014101 (2011)

[22] G.A. Edwards, K. Stiller, G.L. Dunlop, et al., Acta Mater 46, 3893 (1998)

[23] M. Murayama, K. Hono, Acta Mater 47, 1537 (1999)

[24] A. Serizawa, S. Hirosawa, T. Sato, Metall Mater Trans A 39A, 243 (2008)

[25] V. Fallah, A. Korinek, N. Ofori-Opoku, et al., 82, (2015)

[26] C. Marioara, S.J. Andersen, J. Friis, et al., in: International Conference on Aluminium Alloys (ICAA16), M. Wells, M. Brochu (Eds.) Canadian Institute of Mining, Metallurgy \& Petroleum, Montréal, 2018, p. 403435

[27] D. Petschke, F. Lotter, E. Bläss, et al., J Appl Cryst 51, 1339 (2018)

[28] M.W. Zandbergen, Q. Xu, A. Cerezo, et al., Acta Mater 101, $136(2015)$

[29] F. de Geuser, B. Gault, Acta Mater 188, 406 (2020)

[30] A. Somoza, A. Dupasquier, I.J. Polmear, et al., Phys Rev B 61, 14464 (2000)

[31] R. Ferragut, A. Somoza, A. Dupasquier, J Phys-Condens Mat 10, 3903 (1998)

[32] A.A. Istratov, O.F. Vyvenko, Rev Sci Instrum. 70, 1233 (1999)
[33] B. Somieski, T.E.M. Staab, R. Krause-Rehberg, Nucl Instrum Meth A 381, 128 (1996)

[34] T.E.M. Staab, B. Somieski, R. Krause-Rehberg, Nucl Instrum Meth A 381, 141 (1996)

[35] M. Liu, J. Čížek, C.S.T. Chang, et al., Acta Mater 91, 355 (2015)

[36] M. Saga, Y. Sasaki, M. Kikuchi, et al., Mater Sci Forum 217, 821 (1996)

[37] I. Kovács, J. Lendvai, E. Nagy, Acta Metall 20, 975 (1972)

[38] A. Serizawa, T. Sato, in: 11th International Conference on Aluminium Alloys (ICAA11), J. Hirsch, B. Skrotzki, G. Gottstein (Eds.) Wiley VCH, Aachen, Germany, 2008, pp. 915-921

[39] S. Pogatscher, H. Antrekowitsch, H. Leitner, et al., Acta Mater 60, 4496 (2012)

[40] J.D. Bryant, Metall Mater Trans A 30A, 1999 (1999)

[41] K. Yamada, T. Sato, A. Kamio, Mater Sci Forum 331-337, $669(2000)$

[42] A. Poznak, R.K.W. Marceau, P.G. Sanders, Mat Sci Eng A 721, 47 (2018)

[43] Z. Yang, Z.Q. Liang, D. Leyvraz, et al., Materialia 7, 100413 (2019)

[44] Z. Yang, PhD thesis, Technische Universität Berlin, Berlin, (2019)

[45] C. Haase, H. Wurst, Z Metallkde 33, 399 (1941) [in German]

[46] F. Federighi, Acta Mater 6, 379 (1958)

[47] R.W. Siegel, J Nucl Mat 69-70, 117 (1978)

[48] E. Povoden-Karadeniz, Therodynamic and diffusion databases of program Matcalc (mc_al_v2.032.tdb mc_al_v2.004.ddb), Vienna (2014).

[49] M. Mantina, Y. Wang, L.Q. Chen, et al., Acta Mater 57, 4102 (2009)

[50] F.D. Fischer, J. Svoboda, F. Appel, et al., Acta Mater 59, 3463 (2011)

[51] M.J. Starink, L.F. Cao, P.A. Rometsch, Acta Mater 60, 4194 (2012)

[52] Y. Yan, PhD thesis, Technische Universität Berlin, Berlin, (2014) [doi: 10.14279/depositonce-4020]

[53] M. Madanat, M. Liu, X.-P. Zhang, et al., Phys Rev Mater 4, 063608 (2020)

[54] A. Hoell, I. Zizak, H. Bieder, et al., Patent DE 102006029 449, 2006.

[55] J. Wernecke, C. Gollwitzer, P. Müller, et al., J. Synchrotron Rad., 529 (2014)

[56] R.N. Lumley, I.J. Polmear, A.J. Morton, Mater Sci TechLond 21, 1025 (2005)

[57] X.P. Zhang, PhD thesis, Technische Universität Berlin, Berlin, (2019) [doi: 10.14279/depositonce-9247]

[58] F.A. Martinsen, F.J.H. Ehlers, M. Torsæter, et al., Acta Mater 60, 6091 (2012)

[59] J.H. Kim, E. Kobayashi, T. Sato, Mater Trans 56, 1771 (2015)

[60] A. Röhsler, Diploma thesis, Bergakademie Freiberg, Freiberg, (2015)

[61] P.E. Fortin, Can Metall Quart 2, 143 (1963)

[62] S. Muromachi, T. Mae, J Jap Inst Metals Mater 38, 130 (1974) [in Japanese]

[63] K. Yamada, A. Sato, A. Kamio, J Jap Inst Light Met 51, 215 (2001) [in Japanese]

[64] J. Røyset, T. Stene, J.A. Sæter, et al., Mater Sci Forum 519-521, 239 (2006)

[65] L.Z. He, H.-T. Zhang, J.-H. Cui, J Mater Sci Technol 26, $141(2010)$

[66] S. Wenner, C.D. Marioara, S.J. Andersen, et al., Int J Mater Res 103, 948 (2012)

[67] H. Borchers, M. Kainz, Z Metallkde 52, 685 (1961) [in German] 